\title{
EXISTENCE RESULTS FOR RIEMANN-LIOUVILLE FRACTIONAL EVOLUTION INCLUSIONS
}

\author{
YONG HUANG, JINGYUN LV, AND ZHENHAI LIU
}

Received 01 October, 2014

\begin{abstract}
In recent years, differential inclusions with fractional order have been extensively studied by many authors. However, up to now fractional evolution inclusions with RiemannLiouville derivative have not been considered in the literature. In order to fill this gap, in this paper we establish sufficient conditions for the existence of solutions of fractional evolution inclusions involving the Riemann-Liouville fractional derivative. The cases of convex-valued and nonconvex-valued right-hand sides are considered and we present a version of Filippov's theorem for fractional semilinear differential inclusions with Riemann-Liouville derivative.
\end{abstract}

2010 Mathematics Subject Classification: 34B10; 34B15

Keywords: fractional evolution inclusions, Riemann-Liouville fractional derivatives, mild solutions, multivalued map

\section{INTRODUCTION}

Due to the fact that fractional order derivatives and integrals have extensive applications in viscoelasticity, analytical chemistry, electromagnetic, neuron modeling and biological sciences, the theory of fractional calculus has attracted great interest from the mathematical science research community. There has been a significant development in fractional differential equations in recent years, see the monographs of Samako et al. [14], Kilbas et al. [13], Miller and Ross [20], Podlubny [22] and the references therein.

Differential inclusions arise in the mathematical modelling of certain problems in economics, optimal control, stochastic analysis, etc. and are widely investigated by many authors, see $[1,4,15,17,18]$ and references therein.

Recently, fractional differential equations and inclusions with Caputo and RiemannLiouville fractional derivative with different conditions were studied by many authors.

The first author was supported by NSF of Guangxi Grant Nos. 2013GXNSFAA019022 and Scientific Research Fund of Guangxi Education Department Grant No.2013YB243. The second and third authors were supported by NNSF of China Grant Nos.11271087, 61263006, NSF of Guangxi Grant No. 2014GXNSFDA118002, Special Funds of Guangxi Distinguished Experts Construction Engineering. 
In [10], J. Henderson and A. Ouahab investigated the existence of solutions for fractional differential inclusions with infinite delay

$$
\left\{\begin{array}{l}
{ }^{L} D^{\alpha} x(t) \in F\left(t, x_{t}\right), t \in J:=[0, b], 0<\alpha<1, \\
x(t)=\phi(t), t \in[-r, 0]
\end{array}\right.
$$

where ${ }^{L} D^{\alpha}$ is the Riemann-Liouville fractional derivative, $F: J \times C([-r, 0], R) \rightarrow$ $\mathcal{P}(R)$ is a multivalued map with compact values, $\mathcal{P}(R)$ is the family of all nonempty subsets of $R, \phi \in C([-r, 0], R)$ and $\phi(0)=0 . \quad y_{t}$ is the element of $C([-r, 0], R)$ defined by $y_{t}(\theta)=y(t+\theta), \theta \in[-r, 0]$.

In [21], A. Ouahab studied the existence of solutions for fractional semilinear differential inclusions of the form

$$
\left\{\begin{array}{l}
\left({ }^{c} D^{\alpha} x-A x\right)(t) \in F(t, x(t)), \text { a.e. } t \in J:=[0, b], 0<\alpha<1, \\
x(0)=x_{0} \in X,
\end{array}\right.
$$

where ${ }^{c} D^{\alpha}$ is the Caputo fractional derivative, $F: J \times X \rightarrow \mathcal{P}(X)$ is a multivalued map, $A$ is an almost sectorial operator and $X$ is a sparable Banach space.

In [25], Y. Zhou, L. Zhang and X.H. Shen researched the existence of solutions for fractional evolution equation with Riemann-Liouville fractional derivative of the form

$$
\left\{\begin{array}{l}
{ }^{L} D^{q} x(t)=A x(t)+(F x)(t), t \in J^{\prime}:=(0, a], 0<q<1, \\
\left(I_{0^{+}}^{1-q} x\right)(0)+g(x)=x_{0}
\end{array}\right.
$$

where ${ }^{L} D^{\alpha}$ is the Riemann-Liouville fractional derivative of order $q, I_{0^{+}}^{1-q}$ is Riemann-Liouville integral of order $1-q, A$ is the infinitesimal generator of a $C_{0^{-}}$semigroup $\{T(t), t \geq 0\}$ on a Banach space $X . F: C\left(J^{\prime}, X\right) \rightarrow L\left(J^{\prime}, X\right)$ and $g$ : $C\left(J^{\prime}, X\right) \rightarrow L\left(J^{\prime}, X\right)$ are given functions satisfying some assumptions.

Motivated by the above work, the goal of the present paper is to study the existence of solutions for fractional evolution inclusions with Riemann-Liouville fractional derivative of the form

$$
\left\{\begin{array}{l}
L_{D_{0+}^{\alpha}}^{\alpha} x(t) \in A x(t)+F(t, x(t)), \text { a.e. } t \in(0, b], 0<\alpha<1, \\
\left.I_{0^{+}}^{1-\alpha} x(t)\right|_{t=0}=x_{0} \in X
\end{array}\right.
$$

where ${ }^{L} D_{0+}^{\alpha}$ is the Riemann-Liouville fractional derivative of order $\alpha$ with the lower limit zero, $I_{0^{+}}^{1-\alpha}$ is Riemann-Liouville integral of order $1-\alpha$. $A: D(A) \subseteq X \rightarrow X$ is the infinitesimal generator of a $C_{0}$-semigroup $\{T(t), t \geq 0\}$ on a Banach space $X$. $F: J \times X \rightarrow \mathcal{P}(X):=2^{X} \backslash\{\varnothing\}$ is a multivalued map satisfying some assumptions and $J=[0, b]$.

The existence of mild solutions for the fractional evolution inclusions with Caputo fractional derivative has been researched in several recent papers (see [19, 21, 23]), much less is known about the fractional evolution inclusions with Riemann-Liouville fractional derivative. In this paper, our goal is to give the existence results in both convex and nonconvex cases for (1.1) and present a version of Filippov's theorem. 
The results we obtained are a generalization and continuation of the recent results on this issue.

The rest of this paper is organized as follows. In Section 2, some notations and preparation results are given. In Section 3, using the nonlinear alternative of LeraySchauder, we present an existence result for problem (1.1) in the case where the right-hand side is convex-valued. In Section 4, we use a fixed point theorem for contraction multivalued maps to (1.1), for the nonconvex case. In Section 5, we shall be concerned with Filippov's theorem for (1.1). In the end, we present an example to demonstrate our main results in Section 6.

\section{PReliminaries}

In this section, we introduce the notations, definitions, and preliminary facts that will be used in the remainder of this paper.

Let $C(J, X)$ denotes the Banach space of all $\mathrm{X}$-value continuous functions from $J=[0, b]$ into $X$ with the norm $\|x\|_{C(J, X)}=\sup _{t \in J}\|x(t)\|$. For measurable functions $m: J \rightarrow R$, we define the norm $\|m\|_{L^{P}(J, R)}:=\left(\int_{J}|m(t)|^{p} d t\right)^{\frac{1}{p}}, 1 \leq p<\infty$. $L^{p}(J, R)(1 \leq p<\infty)$ be the Banach space of all Lebesgue measurable functions from $J$ into $R$ with $\|m\|_{L^{P}(J, R)}<\infty$. Let $L^{p}(J, X)$ be the Banach space of functions $m: J \rightarrow X$ which are Bochner integrable normed by $\|m\|_{L^{P}(J, X)}$. Throughout this paper, we suppose $M:=\sup _{t \in[0, \infty)}\|T(t)\|<\infty$. Let $J^{\prime}=(0, b]$, to define the mild solutions of (1.1), we also consider the Banach space $C_{1-\alpha}(J, X)=\{x \in$ $\left.C\left(J^{\prime}, X\right): t^{1-\alpha} x(t) \in C(J, X)\right\}$ with the norm $\|x\|_{C_{1-\alpha}}=\sup _{t \in J}\left\{t^{1-\alpha}\|x(t)\|_{X}\right\}$. It is easy to see $\left(C_{1-\alpha}(J, X),\|\cdot\|_{C_{1-\alpha}}\right)$ is a Banach space.

First, let us recall the following definitions from fractional calculus. For more details, one can see $[13,20,22]$.

Definition 1. The fractional integral of order $q$ with the lower limit zero for a function $f$ is defined as

$$
I_{0^{+}}^{q} f(t)=\frac{1}{\Gamma(q)} \int_{0}^{t} \frac{f(s)}{(t-s)^{1-q}} d s, t>0, q>0,
$$

provided the right side is point-wise defined on $[0, \infty)$, where $\Gamma(\cdot)$ is the gamma function.

Definition 2. The Riemann-Liouville derivative of order $q$ with the lower limit zero for a function $f:[0, \infty) \rightarrow R$ can be written as

$$
{ }^{L} D_{0+}^{q} f(t)=\frac{1}{\Gamma(n-q)}\left(\frac{d}{d t}\right)^{n} \int_{0}^{t} \frac{f(s)}{(t-s)^{q-n+1}} d s, t>0, n-1<q<n .
$$


Definition 3. The Caputo derivative of order $q$ for a function $f:[0, \infty) \rightarrow R$ can be written as

$$
{ }^{c} D_{0^{+}}^{q} f(t)={ }^{L} D^{q}\left[f(t)-\sum_{k=0}^{n-1} \frac{t^{k}}{k !} f^{(k)}(0)\right], t>0, n-1<q<n .
$$

Remark 1. (i) If $f(t) \in C^{n}[0, \infty)$, then

$$
{ }^{c} D_{0^{+}}^{q} f(t)=\frac{1}{\Gamma(n-q)} \int_{0}^{t} \frac{f^{(n)}(s)}{(t-s)^{q+1-n}} d s=I_{0^{+}}^{n-q} f^{(n)}(t), t>0, n-1<q<n .
$$

(ii) The Caputo derivative of a constant is equal to zero.

(i i i ) If $f$ is an abstract function with values in $X$, then integrals which appear in Definition 1 and 2 are taken in Bochner's sense.

Now, we also introduce some basic definitions on multivalued maps. For more details, see [2,7,12].

We use the notations:

$$
\mathcal{P}_{c l}(X)=\{Y \in \mathcal{P}(X): Y \text { is closed }\}, \mathcal{P}_{b}(X)=\{Y \in \mathcal{P}(X): Y \text { is bounded }\},
$$$$
\mathcal{P}_{c v}(X)=\{Y \in \mathcal{P}(X): Y \text { is convex }\}, \mathcal{P}_{c p}(X)=\{Y \in \mathcal{P}(X): Y \text { is compact }\} \text {. }
$$

A multivalued map $G: X \rightarrow \mathcal{P}(X)$ is convex (closed) valued if $G(x)$ is convex (closed) for all $x \in X$. $G$ is bounded on bounded sets if $G(B)=\bigcup_{x \in B} G(x)$ is bounded in $X$ for any bounded set $B$ of $X$, i.e., $\sup _{x \in B}\{\sup \{\|y\|: y \in G(x)\}\}<\infty$.

$\mathrm{G}$ is called upper semicontinuous (u.s.c.) on $X$ if for each $x_{0} \in X$, the set $G\left(x_{0}\right)$ is a nonempty closed subset of $X$, and if for each open set $U$ of $X$ containing $G\left(x_{0}\right)$, there exists an open neighborhood $V$ of $x_{0}$ such that $G(V) \subseteq U$.

$G$ is said to be completely continuous if $G(B)$ is relatively compact for every $B \in \mathcal{P}_{b}(X)$.

If the multivalued map $G$ is completely continuous with nonempty compact values, then $G$ is u.s.c. if and only if $G$ has a closed graph (i.e., $x_{n} \rightarrow x_{*}, y_{n} \rightarrow y_{*}, y_{n} \in$ $G\left(x_{n}\right)$ imply $\left.y_{*} \in G\left(x_{*}\right)\right)$.

A multivalued map $G: J \rightarrow \mathcal{P}_{c l}(X)$ is said to be measurable if for each $x \in X$ the function $Y: J \rightarrow R^{+}$defined by $Y(t)=d(x, G(t))=\inf \{\|x-z\|: z \in G(t)\}$ is measurable.

Let $(X, d)$ be a metric space induced from the normed space $(X,\|\cdot\|)$. Consider $H_{d}: \mathcal{P}(X) \times \mathcal{P}(X) \rightarrow R_{+} \cup\{\infty\}$, given by

$$
H_{d}(A, B)=\max \left\{\sup _{a \in A} d(a, B), \sup _{b \in B} d(b, A)\right\},
$$

where $d(a, B)=\inf _{b \in B} d(a, b), d(b, A)=\inf _{a \in A} d(a, b)$.

Then $\left(\mathcal{P}_{b, c l}(X), H_{d}\right)$ is a metric space and $\left(\mathcal{P}_{c l}(X), H_{d}\right)$ is a generalized metric space (see [15]).

Definition 4 ([6]). A multivalued operator $G: X \rightarrow \mathcal{P}_{c l}(X)$ is called 
(1) $\gamma$-Lipschitz if and only if there exists $\gamma>0$ such that

$$
H_{d}(G(x), G(y)) \leq \gamma d(x, y), \text { for each } x, y \in X,
$$

(2) a contraction if and only if it is $\gamma$-Lipschitz with $\gamma<1$.

Lemma 1 ([6]). Let $(X, d)$ be a complete metric space. If $G: X \rightarrow \mathcal{P}_{c l}(X)$ is a contraction, then Fix $G \neq \varnothing$.

Lemma 2 ([8]). Let $X$ be a separable complete metric space and let $G:[0, b] \rightarrow$ $\mathcal{P}(X)$ be a measurable multivalued map with nonempty closed values. Then $G$ has a measurable selection.

Lemma 3 ([26]). Let $G:[0, b] \rightarrow \mathcal{P}(X)$ be a measurable multivalued map and $u:[0, b] \rightarrow X$ a measurable function. Then for any measurable $v:[0, b] \rightarrow(0,+\infty)$ there exist a measurable selection $f_{v}$ of $G$ such that for a.e. $t \in[0, b]$,

$$
\left\|u(t)-f_{v}(t)\right\| \leq d(u(t), G(t))+v(t) .
$$

Lemma 4. Let $G:[0, b] \rightarrow \mathcal{P}_{c p}(X)$ be a measurable multivalued map and $u:$ $[0, b] \rightarrow X$ a measurable function. Then there exists a measurable selection $f$ of $G$ such that for a.e. $t \in[0, b]$,

$$
\|u(t)-f(t)\| \leq d(u(t), G(t)) .
$$

Lemma 5 (Lasota and Opial [16]). Let $J$ be a compact real interval and $E$ be a Banach space. The multivalued map $F: J \times E \rightarrow \mathcal{P}_{b, c l, c v}(E)$ is measurable to $t$ for each fixed $x \in E$, u.s.c. to $x$ for a.e. $t \in J$, and for each $x \in C(J, E)$ the set $S_{F, x}=\left\{f \in L^{1}(J, E): f(t) \in F(t, x(t))\right.$, a.e. $\left.t \in J\right\}$ is nonempty. Let $\Gamma$ be a linear continuous mapping from $L^{1}(J, E)$ to $C(J, E)$, then the operator

$$
\Gamma \circ S_{F}: C(J, E) \rightarrow \mathcal{P}_{b, c l, c v}(C(J, E)), x \mapsto\left(\Gamma \circ S_{F}\right)(x)=\Gamma\left(S_{F, x}\right),
$$

is a closed graph operator in $C(J, E) \times C(J, E)$.

Lemma 6 (Nonlinear alternative for Kakutani maps [9]). Let $E$ be a Banach space, $C$ a closed convex subset of $E$. $U$ an open subset of $C$ and $0 \in U$. Suppose that $G: \bar{U} \rightarrow \mathcal{P}_{c p, c v}(E)$ is an upper semicontinuous compact map. Then either:

(i) $G$ has a fixed point in $\bar{U}$, or

(ii) there is $u \in \partial U$ and $\lambda \in(0,1)$ with $u \in \lambda G(u)$.

On the basis of [25], we give the following definition of mild solution of system (1.1).

Definition 5. A function $x \in C_{1-\alpha}(J, X)$ is called a mild solution of (1.1) if $\left.I_{0^{+}}^{1-\alpha} x(t)\right|_{t=0}=x_{0}$ and there exists $f \in L^{1}(J, X)$ such that $f(t) \in F(t, x(t))$ for a.e. $t \in J$ and

$$
x(t)=t^{\alpha-1} T_{\alpha}(t) x_{0}+\int_{0}^{t}(t-s)^{\alpha-1} T_{\alpha}(t-s) f(s) d s, t \in(0, b]
$$


where

$$
T_{\alpha}(t)=\alpha \int_{0}^{\infty} \theta M_{\alpha}(\theta) T\left(t^{\alpha} \theta\right) d \theta,
$$

and $M_{\alpha}$ is a probability density function which is defined by

$$
M_{\alpha}(\theta)=\sum_{n=1}^{\infty} \frac{(-\theta)^{n-1}}{(n-1) ! \Gamma(1-\alpha n)}, 0<\alpha<1, \theta \in \mathbb{C} .
$$

Lemma 7 ([24]). The operator $T_{\alpha}(t)$ has the following properties:

(i) For any fixed $t \geq 0, T_{\alpha}(t)$ is linear and bounded operators, i.e., for any $x \in X$,

$$
\left\|T_{\alpha}(t) x\right\| \leq \frac{M}{\Gamma(\alpha)}\|x\| .
$$

(ii) $T_{\alpha}(t)(t \geq 0)$ is strongly continuous.

(i i i ) For every $t>0, T_{\alpha}(t)$ is also compact operator if $T(t)$ is compact.

Now we state the following generalization of Gronwall's lemma for singular kernels whose proof can be found in ([11], Lemma 7.1.1). This will be essential for the main result of Section 3.

Lemma 8. Let $v:[0, b] \rightarrow[0, \infty)$ be a real function and $\omega(\cdot)$ is a nonnegative, locally integrable function on $[0, b]$ and there are constants $a>0$ and $0<\alpha<1$ such that

$$
v(t) \leq \omega(t)+a \int_{0}^{t}(t-s)^{-\alpha} v(s) d s .
$$

Then there exists a constant $K=K(\alpha)$ such that

$$
v(t) \leq \omega(t)+K a \int_{0}^{t}(t-s)^{-\alpha} \omega(s) d s,
$$

for every $t \in[0, b]$.

\section{EXISTENCE OF MILD SOLUTIONS FOR CONVEX CASE}

Assume in this section that $F: J \times X \rightarrow \mathcal{P}(X)$ is a convex valued multivalued map. Before stating and proving the main results, we introduce the following hypotheses.

(1) $T(t)$ is a compact operator for every $t>0$.

(2) $F: J \times X \rightarrow \mathcal{P}_{c p, c v}(X)$ is a multivalued map such that

(a) for each fixed $u \in X$, the map $t \mapsto F(t, u)$ is measurable,

(b) for a.e. $t \in J$, the map $u \mapsto F(t, u)$ is u.s.c.,

(c) for each $x \in C_{1-\alpha}(J, X)$ the set

$$
S_{F, x}=\left\{f \in L^{1}(J, X): f(t) \in F(t, x(t))\right\}
$$

is nonempty. 
(3) There exists a function $\phi(t) \in L^{\frac{1}{q}}\left(J, R^{+}\right), q \in(0, \alpha)$ and a constant $c>0$ such that

$$
\begin{aligned}
& \|F(t, x(t))\|_{X}=\sup \left\{\|f(t)\|_{X}: f(t) \in F(t, x(t))\right\} \leq \phi(t)+c t^{1-\alpha}\|x(t)\|, \\
& \quad \text { for a.e. } t \in J \text { and all } x \in C_{1-\alpha}(J, X) .
\end{aligned}
$$

Theorem 1. Assume that hypotheses (1) - (3) hold, then system (1.1) has at least one mild solution.

Proof. Consider the multivalued operator $N: C_{1-\alpha}(J, X) \rightarrow \mathcal{P}\left(C_{1-\alpha}(J, X)\right)$ defined by

$N(x)=\left\{\begin{array}{l}h \in C_{1-\alpha}(J, X): \\ h(t)=t^{\alpha-1} T_{\alpha}(t) x_{0}+\int_{0}^{t}(t-s)^{\alpha-1} T_{\alpha}(t-s) f(s) d s, f \in S_{F, x}, t \in J^{\prime}\end{array}\right\}$.

Using (1) - (3) and Lemma 7, one can easily prove that $N(x) \subseteq C_{1-\alpha}(J, X)$ for any $x \in C_{1-\alpha}(J, X)$.

Clearly, the fixed points of the operator $N$ are mild solutions of problem (1.1). We shall show that $N$ satisfies all conditions of Lemma 6. The proof will be given in several steps.

Step 1: $N(x)$ is convex for each $x \in C_{1-\alpha}(J, X)$.

Since $S_{F, x}$ is convex (because $F$ has convex values) then one can easily show that $N(x)$ is convex for each $x \in C_{1-\alpha}(J, X)$.

Step 2: $N$ maps bounded sets into relatively compact sets in $C_{1-\alpha}(J, X)$. Define

$$
\begin{gathered}
B_{r}=\left\{x \in C_{1-\alpha}(J, X):\|x\|_{C_{1-\alpha}} \leq r\right\}, \\
\Omega=\left\{y \in C(J, X): y(t)=t^{1-\alpha} h(t), h \in N\left(B_{r}\right)\right\}
\end{gathered}
$$

We shall show that $N\left(B_{r}\right)$ is relatively compact set. We subdivide the proof into several claims.

Claim 1: $\Omega$ is a bounded set of $C(J, X)$.

Let $x \in B_{r}$ and $h \in N(x)$, then there exists $f \in S_{F, x}$ such that, for each $t \in J$, we have

$$
y(t)=t^{1-\alpha} h(t)=T_{\alpha}(t) x_{0}+t^{1-\alpha} \int_{0}^{t}(t-s)^{\alpha-1} T_{\alpha}(t-s) f(s) d s .
$$

Taking into account the imposed assumptions, applying Lemma 7 and Hölder inequality, we obtain

$$
\begin{aligned}
\|y(t)\| & \leq \frac{M}{\Gamma(\alpha)}\left\|x_{0}\right\|+\frac{M t^{1-\alpha}}{\Gamma(\alpha)} \int_{0}^{t}(t-s)^{\alpha-1}\|f(s)\| d s \\
& \leq \frac{M}{\Gamma(\alpha)}\left\|x_{0}\right\|+\frac{M t^{1-\alpha}}{\Gamma(\alpha)} \int_{0}^{t}(t-s)^{\alpha-1}\left[\phi(s)+c s^{1-\alpha}\|x(s)\|\right] d s \\
& \leq \frac{M}{\Gamma(\alpha)}\left\|x_{0}\right\|+\frac{M}{\Gamma(\alpha)}\left(\frac{1-q}{\alpha-q} b\right)^{1-q}\|\phi\|_{L^{\frac{1}{q}(J, X)}}+\frac{c M b r}{\Gamma(\alpha+1)}:=l .
\end{aligned}
$$


Thus, for each $y \in \Omega$, we have $\|y\|_{C(J, X)} \leq l$.

Claim 2: $\Omega \subseteq C(J, X)$ is equicontinuous.

For any $y \in \Omega$, let $0=t_{1}<t_{2} \leq b$, by using (3), Lemma 7 and Holder inequality, we get

$$
\begin{aligned}
\left\|y\left(t_{2}\right)-y(0)\right\| \leq & \left\|T_{\alpha}\left(t_{2}\right) x_{0}-T_{\alpha}(0) x_{0}\right\|+\left\|t_{2}^{1-\alpha} \int_{0}^{t_{2}}\left(t_{2}-s\right)^{\alpha-1} T_{\alpha}\left(t_{2}-s\right) f(s) d s\right\| \\
\leq & \left\|T_{\alpha}\left(t_{2}\right) x_{0}-T_{\alpha}(0) x_{0}\right\|+\frac{M}{\Gamma(\alpha)}\left(\frac{1-q}{\alpha-q} t_{2}\right)^{1-q}\|\phi\|_{L^{\frac{1}{q}}(J, X)} \\
& +\frac{M c r t_{2}}{\Gamma(\alpha+1)} \rightarrow 0, \text { as } t_{2} \rightarrow 0^{+} .
\end{aligned}
$$

For $0<t_{1}<t_{2} \leq b$, selecting $\varepsilon>0$ sufficiently small and taking into account the imposed assumptions, we have

$$
\begin{aligned}
\left\|y\left(t_{2}\right)-y\left(t_{1}\right)\right\| \leq & \left\|T_{\alpha}\left(t_{2}\right) x_{0}-T_{\alpha}\left(t_{1}\right) x_{0}\right\|+\left\|t_{2}^{1-\alpha} \int_{t_{1}}^{t_{2}}\left(t_{2}-s\right)^{\alpha-1} T_{\alpha}\left(t_{2}-s\right) f(s) d s\right\| \\
& +\left\|\int_{0}^{t_{1}}\left[t_{2}^{1-\alpha}\left(t_{2}-s\right)^{\alpha-1}-t_{1}^{1-\alpha}\left(t_{1}-s\right)^{\alpha-1}\right] T_{\alpha}\left(t_{2}-s\right) f(s) d s\right\| \\
& +\left\|t_{1}^{1-\alpha} \int_{0}^{t_{1}}\left(t_{1}-s\right)^{\alpha-1}\left[T_{\alpha}\left(t_{2}-s\right)-T_{\alpha}\left(t_{1}-s\right)\right] f(s) d s\right\| \\
\leq & \left\|T_{\alpha}\left(t_{2}\right) x_{0}-T_{\alpha}\left(t_{1}\right) x_{0}\right\|+\frac{M b^{1-\alpha}}{\Gamma(\alpha)} \int_{t_{1}}^{t_{2}}\left(t_{2}-s\right)^{\alpha-1}[\phi(s)+c r] d s \\
& +\frac{M}{\Gamma(\alpha)} \int_{0}^{t_{1}}\left[t_{1}^{1-\alpha}\left(t_{1}-s\right)^{\alpha-1}-t_{2}^{1-\alpha}\left(t_{2}-s\right)^{\alpha-1}\right](\phi(s)+c r) d s \\
& +\left\|\int_{0}^{t_{1}-\varepsilon} t_{1}^{1-\alpha}\left(t_{1}-s\right)^{\alpha-1}\left[T_{\alpha}\left(t_{2}-s\right)-T_{\alpha}\left(t_{1}-s\right)\right] f(s) d s\right\| \\
& +\left\|\int_{t_{1}-\varepsilon}^{t_{1}} t_{1}^{1-\alpha}\left(t_{1}-s\right)^{\alpha-1}\left[T_{\alpha}\left(t_{2}-s\right)-T_{\alpha}\left(t_{1}-s\right)\right] f(s) d s\right\| \\
\leq & I_{1}+I_{2}+I_{3}+I_{4}+I_{5},
\end{aligned}
$$

where

$$
\begin{gathered}
I_{1}=\left\|T_{\alpha}\left(t_{2}\right) x_{0}-T_{\alpha}\left(t_{1}\right) x_{0}\right\|, \\
I_{2}=\frac{M b^{1-\alpha}}{\Gamma(\alpha)} \int_{t_{1}}^{t_{2}}\left(t_{2}-s\right)^{\alpha-1}[\phi(s)+c r] d s, \\
I_{3}=\frac{M}{\Gamma(\alpha)} \int_{0}^{t_{1}}\left[t_{1}^{1-\alpha}\left(t_{1}-s\right)^{\alpha-1}-t_{2}^{1-\alpha}\left(t_{2}-s\right)^{\alpha-1}\right](\phi(s)+c r) d s, \\
I_{4}=\sup _{s \in\left[0, t_{1}-\varepsilon\right]}\left\|T_{\alpha}\left(t_{2}-s\right)-T_{\alpha}\left(t_{1}-s\right)\right\|\left[\left(\frac{1-q}{\alpha-q} b\right)^{1-q}\|\phi\|_{L^{\frac{1}{q}(J, X)}}+\frac{b c r}{\alpha}\right],
\end{gathered}
$$




$$
I_{5}=\frac{2 M b^{1-\alpha}}{\Gamma(\alpha)} \int_{t_{1}-\varepsilon}^{t_{1}}\left(t_{1}-s\right)^{\alpha-1}[\phi(s)+c r] d s .
$$

According to Lemma 7(ii), it is easy to see that $I_{1} \rightarrow 0$ as $t_{2} \rightarrow t_{1}$. Appling the absolute continuity of the Lebesgue integral, we have $I_{2}$ and $I_{5}$ tend to zero independently of $x \in B_{r}$ as $t_{2} \rightarrow t_{1}, \varepsilon \rightarrow 0$. Noting that

$0<\left[t_{1}^{1-\alpha}\left(t_{1}-s\right)^{\alpha-1}-t_{2}^{1-\alpha}\left(t_{2}-s\right)^{\alpha-1}\right](\phi(s)+c r)<t_{1}^{1-\alpha}\left(t_{1}-s\right)^{\alpha-1}(\phi(s)+c r)$, and $\int_{0}^{t_{1}} t_{1}^{1-\alpha}\left(t_{1}-s\right)^{\alpha-1}(\phi(s)+c r) d s$ exists, then by the Lebesgue dominated convergence theorem, we have $I_{3}$ tends to zero independently of $x \in B_{r}$ as $t_{2} \rightarrow t_{1}$. Since (1) and Lemma 7 imply that the continuity of $T_{\alpha}(t)(t>0)$ in $t$ in the uniform operator topology, it is easy to see that $I_{4}$ tends to zero independently of $x \in B_{r}$ as $t_{2} \rightarrow t_{1}$. Thus, $\left\|y\left(t_{2}\right)-y\left(t_{1}\right)\right\|$ tends to zero independently of $x \in B_{r}$ as $t_{2} \rightarrow t_{1}$, which means that $\Omega$ is equicontinuous.

Claim 3: For any $t \in J, \Omega(t)$ is relatively compact in $X$.

This is trivial for $t=0$, since $\Omega(0)=\left\{\frac{x_{0}}{\Gamma(\alpha)}\right\}$. So it is only necessary to consider $0<t \leq b$. Let $0<t \leq b$ be fixed. For $x \in B_{r}$ and $h \in N(x)$ there exists $f \in S_{F, x}$ such that

$$
y(t)=t^{1-\alpha} h(t)=T_{\alpha}(t) x_{0}+t^{1-\alpha} \int_{0}^{t}(t-s)^{\alpha-1} T_{\alpha}(t-s) f(s) d s .
$$

For $\forall \varepsilon \in(0, t), \forall \delta>0$, define

$$
\begin{aligned}
y^{\varepsilon, \delta}(t)= & \alpha \int_{\delta}^{\infty} \theta M_{\alpha}(\theta) T\left(t^{\alpha} \theta\right) x_{0} d \theta \\
& +\alpha t^{1-\alpha} \int_{0}^{t-\varepsilon} \int_{\delta}^{\infty}(t-s)^{\alpha-1} \theta M_{\alpha}(\theta) T\left((t-s)^{\alpha} \theta\right) f(s) d \theta d s \\
\leq & T\left(\varepsilon^{\alpha} \delta\right)\left\{\alpha \int_{\delta}^{\infty} \theta M_{\alpha}(\theta) T\left(t^{\alpha} \theta-\varepsilon^{\alpha} \delta\right) x_{0} d \theta\right. \\
& \left.+\alpha t^{1-\alpha} \int_{0}^{t-\varepsilon} \int_{\delta}^{\infty}(t-s)^{\alpha-1} \theta M_{\alpha}(\theta) T\left((t-s)^{\alpha} \theta-\varepsilon^{\alpha} \delta\right) f(s) d \theta d s\right\} .
\end{aligned}
$$

Then from the compactness of $T\left(\varepsilon^{\alpha} \delta\right)\left(\varepsilon^{\alpha} \delta>0\right)$, we obtain that the set $\Omega^{\varepsilon, \delta}(t)=$ $\left\{y^{\varepsilon, \delta}(t), y \in \Omega\right\}$ is relatively compact in $X$ for $\forall \varepsilon \in(0, t)$ and $\forall \delta>0$.

Moreover, we have

$$
\begin{aligned}
\left\|y(t)-y^{\varepsilon, \delta}(t)\right\| \leq & \left\|\alpha \int_{0}^{\delta} \theta M_{\alpha}(\theta) T\left(t^{\alpha} \theta\right) x_{0} d \theta\right\| \\
& +\left\|\alpha t^{1-\alpha} \int_{0}^{t} \int_{0}^{\delta}(t-s)^{\alpha-1} \theta M_{\alpha}(\theta) T\left((t-s)^{\alpha} \theta\right) f(s) d \theta d s\right\| \\
& +\left\|\alpha t^{1-\alpha} \int_{t-\varepsilon}^{t} \int_{\delta}^{\infty}(t-s)^{\alpha-1} \theta M_{\alpha}(\theta) T\left((t-s)^{\alpha} \theta\right) f(s) d \theta d s\right\|
\end{aligned}
$$




$$
\begin{aligned}
\leq & \alpha M\left\|x_{0}\right\| \int_{0}^{\delta} \theta M_{\alpha}(\theta) d \theta+\alpha M b^{1-\alpha} \int_{0}^{t}(t-s)^{\alpha-1}[\phi(s)+c r] d s \int_{0}^{\delta} \theta M_{\alpha}(\theta) d \theta \\
& +\alpha M b^{1-\alpha} \int_{t-\varepsilon}^{t}(t-s)^{\alpha-1}[\phi(s)+c r] d s \int_{0}^{\infty} \theta M_{\alpha}(\theta) d \theta \\
\leq & \alpha M\left\|x_{0}\right\| \int_{0}^{\delta} \theta M_{\alpha}(\theta) d \theta+\alpha M b^{1-\alpha} \int_{0}^{t}(t-s)^{\alpha-1}[\phi(s)+c r] d s \int_{0}^{\delta} \theta M_{\alpha}(\theta) d \theta \\
& +\frac{M b^{1-\alpha}}{\Gamma(\alpha)} \int_{t-\varepsilon}^{t}(t-s)^{\alpha-1}[\phi(s)+c r] d s \rightarrow 0, \text { as } \varepsilon \rightarrow 0, \delta \rightarrow 0 .
\end{aligned}
$$

Therefore, there are relatively compact sets arbitrarily close to the set $\Omega(t), t>0$. Hence the set $\Omega(t), t>0$ is also relatively compact in $X$.

As a consequence of Claims 1-3 together with the Arzola-Ascoli theorem, we can conclude that $\Omega \subseteq C(J, X)$ is relatively compact set. Thus, one can easily obtain that $N\left(B_{r}\right)$ is relatively compact set in $C_{1-\alpha}(J, X)$.

Step 3: $\mathrm{N}$ has a closed graph.

For any $x \in C_{1-\alpha}(J, X)$, according to (2), there exists $f \in S_{F, x}$. Set $y(t)=$ $t^{1-\alpha} x(t)$ and define $\widetilde{N}$ as follows

$\widetilde{N}(y)=\left\{\begin{array}{l}\widetilde{h} \in C(J, X): \\ \widetilde{h}(t)=T_{\alpha}(t) x_{0}+t^{1-\alpha} \int_{0}^{t}(t-s)^{\alpha-1} T_{\alpha}(t-s) f(s) d s, f \in S_{F, x}, t \in J\end{array}\right\}$.

Taking into account the imposed assumptions, we see that $\widetilde{N}: C(J, X) \rightarrow C(J, X)$.

Let $y_{n} \rightarrow y_{*}(n \rightarrow \infty), \widetilde{h}_{n} \in \widetilde{N}\left(y_{n}\right), \widetilde{h}_{n} \rightarrow \widetilde{h}_{*}(n \rightarrow \infty)$. We shall prove that $\widetilde{h}_{*} \in \widetilde{N}\left(y_{*}\right)$. Since $\widetilde{h}_{n} \in \widetilde{N}\left(y_{n}\right)$, there exists $f_{n} \in S_{F, x_{n}}$, such that, for each $t \in J$,

$$
\widetilde{h}_{n}(t)=T_{\alpha}(t) x_{0}+t^{1-\alpha} \int_{0}^{t}(t-s)^{\alpha-1} T_{\alpha}(t-s) f_{n}(s) d s .
$$

We have to prove that there exists $f_{*} \in S_{F, x_{*}}$ such that, for each $t \in J$,

$$
\widetilde{h}_{*}(t)=T_{\alpha}(t) x_{0}+t^{1-\alpha} \int_{0}^{t}(t-s)^{\alpha-1} T_{\alpha}(t-s) f_{*}(s) d s .
$$

Since $\widetilde{h}_{n} \rightarrow \widetilde{h}_{*}(n \rightarrow \infty)$, we can obtain

$$
\left\|\left(\widetilde{h}_{n}(t)-T_{\alpha}(t) x_{0}\right)-\left(\widetilde{h}_{*}(t)-T_{\alpha}(t) x_{0}\right)\right\| \rightarrow 0, \text { as } n \rightarrow \infty .
$$

Consider the linear continuous operator

$$
\begin{gathered}
\Gamma: L^{\frac{1}{q}}(J, X) \rightarrow C(J, X), \\
(\Gamma f)(t)=t^{1-\alpha} \int_{0}^{t}(t-s)^{\alpha-1} T_{\alpha}(t-s) f(s) d s .
\end{gathered}
$$

Clearly it follows from Lemma 5 that $\Gamma \circ S_{F}$ is a closed graph operator. Moreover, we have

$$
\widetilde{h}_{n}(t)-T_{\alpha}(t) x_{0} \in \Gamma\left(S_{F, x_{n}}\right) .
$$


Since $y_{n} \rightarrow y_{*}$, it follows from Lemma 5 that

$$
\widetilde{h}_{*}(t)-T_{\alpha}(t) x_{0} \in \Gamma\left(S_{F, x_{*}}\right) .
$$

Therefore, $\widetilde{N}$ has a closed graph. Thus, one can easily obtain that $N$ has a closed graph. Since $N$ is a completely continuous multivalued map with compact valued, we have $N$ is u.s.c.

Step 4: A priori bounds on solutions.

Let $x$ be a possible solution of (1.1). Then $x \in N(x)$ and there exists $f \in S_{F, x}$ such that

$$
x(t)=t^{\alpha-1} T_{\alpha}(t) x_{0}+\int_{0}^{t}(t-s)^{\alpha-1} T_{\alpha}(t-s) f(s) d s, t \in J^{\prime} .
$$

Moreover

$$
\begin{aligned}
t^{1-\alpha}\|x(t)\| \leq & \left\|T_{\alpha}(t) x_{0}\right\|+\left\|t^{1-\alpha} \int_{0}^{t}(t-s)^{\alpha-1} T_{\alpha}(t-s) f(s) d s\right\| \\
\leq & \frac{M}{\Gamma(\alpha)}\left\|x_{0}\right\|+\frac{t^{1-\alpha} M}{\Gamma(\alpha)} \int_{0}^{t}(t-s)^{\alpha-1}\left[\phi(s)+c s^{1-\alpha}\|x(s)\|\right] d s \\
\leq & \frac{M}{\Gamma(\alpha)}\left\|x_{0}\right\|+\frac{M}{\Gamma(\alpha)}\left(\frac{1-q}{\alpha-q} b\right)^{1-q}\|\phi\|_{L^{\frac{1}{q}}(J, X)} \\
& +\frac{c b^{1-\alpha} M}{\Gamma(\alpha)} \int_{0}^{t}(t-s)^{\alpha-1} s^{1-\alpha}\|x(s)\| d s
\end{aligned}
$$

Let

$$
v(t)=t^{1-\alpha}\|x(t)\|, \omega(t)=\frac{M}{\Gamma(\alpha)}\left[\left\|x_{0}\right\|+\left(\frac{1-q}{\alpha-q} b\right)^{1-q}\|\phi\|_{L^{\frac{1}{q}}(J, X)}\right],
$$

from Lemma 8, we concluded that there exists a constant $K=K(1-\alpha)$ such that

$$
\begin{aligned}
t^{1-\alpha}\|x(t)\| & \leq \omega(t)+\frac{c b^{1-\alpha} M K}{\Gamma(\alpha)} \int_{0}^{t}(t-s)^{\alpha-1} \omega(s) d s \\
& \leq \frac{M}{\Gamma(\alpha)}\left(1+\frac{c b M K}{\Gamma(\alpha+1)}\right)\left[\left\|x_{0}\right\|+\left(\frac{1-q}{\alpha-q} b\right)^{1-q}\|\phi\|_{L^{\frac{1}{q}}(J, X)}\right]:=\widetilde{M} .
\end{aligned}
$$

Hence

$$
\|x\|_{C_{1-\alpha}} \leq \widetilde{M} .
$$

Let $U=\left\{x \in C_{1-\alpha}(J, X):\|x\|_{C_{1-\alpha}}<\widetilde{M}\right\}$, and consider the operator $N: \bar{U} \rightarrow$ $\mathcal{P}_{c p, c v}(X)$. From the choice of $U$, there is no $x \in \partial U$ such that $x \in \lambda N(x)$ for some $\lambda \in(0,1)$. As a consequence of Lemma 6 , we deduce that $N$ has a fixed point $x$ in $\bar{U}$ which is a mild solution of problem (1.1). The proof is complete. 


\section{EXISTENCE OF MILD SOLUTIONS FOR NONCONVEX CASE}

In this section, we study the case when $F$ not necessarily convex valued. Throughout this section, we assume that $X$ is a separable Banach space.

Let us introduce the following hypotheses.

$\left(\mathcal{A}_{1}\right): F: J \times X \rightarrow \mathcal{P}_{c p}(X)$ is a multivalued map such that

(1) the map $t \mapsto F(t, u)$ is measurable for each $u \in X$,

(2) there exists a function $\rho \in L^{\frac{1}{\gamma}}\left(J, R^{+}\right), \gamma \in(0, \alpha)$ and a constant $d>0$ such that

$$
\|F(t, x(t))\|_{X} \leq \rho(t)+d t^{1-\alpha}\|x(t)\| .
$$

for a.e. $t \in J$ and all $x \in C_{1-\alpha}(J, X)$.

$\left(\mathscr{A}_{2}\right)$ : There exist two functions $l, m \in L^{\frac{1}{\beta}}\left(J, R^{+}\right), \beta \in(0, \alpha)$ such that $H_{d}(F(t, x(t)), F(t, y(t))) \leq l(t)\|x-y\|_{C_{1-\alpha}}$, for a.e. $t \in J$ and all $x, y \in C_{1-\alpha}(J, X)$.

Theorem 2. Assume that $\left(\mathcal{A}_{1}\right)-\left(\mathcal{A}_{2}\right)$ are satisfied. If

$$
\frac{M}{\Gamma(\alpha)}\left(\frac{1-\beta}{\alpha-\beta} b\right)^{1-\beta}\|l\|_{L^{\frac{1}{\beta}(J, X)}}<1,
$$

then problem (1.1) has at least one mild solution.

Proof. Define an operator $N$ on $C_{1-\alpha}(J, X)$ by

$N(x)=\left\{\begin{array}{l}h \in C_{1-\alpha}(J, X): \\ h(t)=t^{\alpha-1} T_{\alpha}(t) x_{0}+\int_{0}^{t}(t-s)^{\alpha-1} T_{\alpha}(t-s) f(s) d s, f \in S_{F, x}, t \in J^{\prime}\end{array}\right\}$.

According to $\left(\mathscr{A}_{1}\right)$ and $\left(\mathscr{A}_{2}\right), N: C_{1-\alpha}(J, X) \rightarrow \mathcal{P}\left(C_{1-\alpha}(J, X)\right)$ is well defined. We will show that $N$ satisfies the assumptions of Lemma 1. The proof is given in two steps.

Step 1: $N(x) \in \mathcal{P}_{c l}\left(C_{1-\alpha}(J, X)\right)$ for each $x \in C_{1-\alpha}(J, X)$. Indeed, let $\left\{h_{n}\right\}_{n \geq 0} \in N(x)$ be such that $h_{n} \rightarrow h$ in $C_{1-\alpha}(J, X)$. Then there exists $f_{n} \in S_{F, x}$ such that

$$
h_{n}(t)=t^{\alpha-1} T_{\alpha}(t) x_{0}+\int_{0}^{t}(t-s)^{\alpha-1} T_{\alpha}(t-s) f_{n}(s) d s, t \in J^{\prime}
$$

According to $\left(\mathcal{A}_{1}\right)$, we infer that for a.e. $t \in J$, we have

$$
\begin{aligned}
\left\|f_{n}(t)\right\|_{X} & \leq \rho(t)+d t^{1-\alpha}\|x(t)\| \\
& \leq \rho(t)+d\|x\|_{C_{1-\alpha}},
\end{aligned}
$$

which implies that $f_{n}$ is bounded in $L^{\frac{1}{\gamma}}(J, X)$, and hence weakly converges to $f$ in $L^{\frac{1}{\gamma}}(J, X)$. Let

$$
h(t)=t^{\alpha-1} T_{\alpha}(t) x_{0}+\int_{0}^{t}(t-s)^{\alpha-1} T_{\alpha}(t-s) f(s) d s, t \in J^{\prime} .
$$


Then, we get

$$
\begin{aligned}
\left\|h_{n}-h\right\|_{C_{1-\alpha}} & =\sup _{t \in J}\left\|t^{1-\alpha} \int_{0}^{t}(t-s)^{\alpha-1} T_{\alpha}(t-s)\left[f_{n}(s)-f(s)\right] d s\right\| \\
& \leq b^{1-\alpha} \sup _{t \in J}\left\|\int_{0}^{t}(t-s)^{\alpha-1} T_{\alpha}(t-s)\left[f_{n}(s)-f(s)\right] d s\right\| .
\end{aligned}
$$

We can easily show that, the mapping $g \rightarrow \int_{0}^{\cdot}(\cdot-s)^{\alpha-1} T_{\alpha}(\cdot-s) g(s) d s: L^{\frac{1}{\nu}}(J, X) \rightarrow$ $C(J, X)$ is compact. Then $\left\|h_{n}-h\right\|_{C_{1-\alpha}} \rightarrow 0$, as $n \rightarrow \infty$, which implies that $h \in$ $N(x)$.

Step 2: There exists $0<\theta<1$ such that

$$
H_{d}(N(x), N(y)) \leq \theta\|x-y\|_{C_{1-\alpha}}, \forall x, y \in C_{1-\alpha}(J, X) .
$$

Let $x, y \in C_{1-\alpha}(J, X)$ and $h \in N(x)$. Then there exists $f \in S_{F, x}$ such that

$$
h(t)=t^{\alpha-1} T_{\alpha}(t) x_{0}+\int_{0}^{t}(t-s)^{\alpha-1} T_{\alpha}(t-s) f(s) d s, t \in J^{\prime} .
$$

According to $\left(\mathscr{A}_{2}\right)$ it follows that

$$
H_{d}(F(t, x(t)), F(t, y(t))) \leq l(t)\|x-y\|_{C_{1-\alpha}} .
$$

Hence there is $\omega \in F(t, y(t))$ such that

$$
\|f(t)-\omega\| \leq l(t)\|x-y\|_{C_{1-\alpha}}, t \in J .
$$

Consider the multivalued map $U: J \rightarrow \mathcal{P}(X)$ given by

$$
U(t)=\left\{x \in X:\|f(t)-x\| \leq l(t)\|x-y\|_{C_{1-\alpha}}\right\} .
$$

The set $V(t)=U(t) \cap F(t, y(t))$ is nonempty since it contains $\omega$. Since the multivalued operator $V$ is measurable with nonempty, closed values (see $[3,5])$, there exists a function $\bar{f}$, which is a measurable selection for $V$. Thus, $\bar{f}(t) \in F(t, y(t))$ and

$$
\|f(t)-\bar{f}(t)\| \leq l(t)\|x-y\|_{C_{1-\alpha}} \text {, for a.e. } t \in J .
$$

Let

$$
\bar{h}(t)=t^{\alpha-1} T_{\alpha}(t) x_{0}+\int_{0}^{t}(t-s)^{\alpha-1} T_{\alpha}(t-s) \bar{f}(s) d s .
$$

Then

$$
\begin{aligned}
\left\|t^{1-\alpha} h(t)-t^{1-\alpha} \bar{h}(t)\right\| & =\left\|t^{1-\alpha} \int_{0}^{t}(t-s)^{\alpha-1} T_{\alpha}(t-s)[f(s)-\bar{f}(s)] d s\right\| \\
& \leq \frac{M t^{1-\alpha}}{\Gamma(\alpha)} \int_{0}^{t}(t-s)^{\alpha-1} l(s)\|x-y\|_{C_{1-\alpha}} d s \\
& \leq \frac{M}{\Gamma(\alpha)}\left(\frac{1-\beta}{\alpha-\beta} b\right)^{1-\beta}\|l\|_{L^{\frac{1}{\beta}(J, X)}}\|x-y\|_{C_{1-\alpha}} .
\end{aligned}
$$


Thus

$$
\|h-\bar{h}\|_{C_{1-\alpha}} \leq \frac{M}{\Gamma(\alpha)}\left(\frac{1-\beta}{\alpha-\beta} b\right)^{1-\beta}\|l\|_{L^{\frac{1}{\beta}(J, X)}}\|x-y\|_{C_{1-\alpha}} .
$$

By an analogous relation, obtained by interchanging the roles of $x$ and $y$, it follows that

$$
H_{d}(N(x), N(y)) \leq \frac{M}{\Gamma(\alpha)}\left(\frac{1-\beta}{\alpha-\beta} b\right)^{1-\beta}\|l\|_{L^{\frac{1}{\beta}}(J, X)}\|x-y\|_{C_{1-\alpha}} .
$$

Thus, $N$ is a contraction. Therefore, applying Lemma 1 we conclude that $N$ has a fixed point $x$, which is a mild solution of problem (1.1). The proof is complete.

\section{FILIPPOV'S THEOREM}

In this section, we assume that $X$ is a separable Banach space.

Let $y_{0} \in X, g \in L^{\frac{1}{q}}(J, X), q \in(0, \alpha)$, and $y \in C_{1-\alpha}(J, X)$ be a mild solution of the following semilinear problem:

$$
\left\{\begin{array}{l}
{ }^{L} D^{\alpha} y(t)=A y(t)+g(t), t \in(0, b], 0<\alpha<1, \\
\left.I_{0^{+}}^{1-\alpha} y(t)\right|_{t=0}=y_{0}
\end{array}\right.
$$

Then

$$
y(t)=t^{\alpha-1} T_{\alpha}(t) y_{0}+\int_{0}^{t}(t-s)^{\alpha-1} T_{\alpha}(t-s) g(s) d s .
$$

We introduce the following hypotheses.

$\left(\mathcal{B}_{1}\right) F: J \times X \rightarrow \mathcal{P}_{c p}(X)$ is a multivalued map such that

(1) for all $u \in X$ the map $t \mapsto F(t, u)$ is measurable,

(2) there exists a function $\sigma(t) \in L^{\frac{1}{q}}\left(J, R^{+}\right), q \in(0, \alpha)$ and a constant $k>0$ such that

$$
\|F(t, x(t))\|_{X}=\sup \left\{\|f(t)\|_{X}: f(t) \in F(t, x(t))\right\} \leq \sigma(t)+k t^{1-\alpha}\|x(t)\|,
$$

a.e. $t \in J$, for all $x \in C_{1-\alpha}(J, X)$

(3) the map $\Lambda: t \rightarrow d\left(g(t), F(t, x(t))\right.$ is $L^{\frac{1}{q}}(q \in(0, \alpha))$ integrable.

$\left(\mathcal{B}_{2}\right)$ There exists $K>0$ such that

$$
H_{d}(F(t, x(t)), F(t, z(t))) \leq K t^{1-\alpha}\|x(t)-z(t)\|, \forall x, z \in C_{1-\alpha}(J, X) .
$$

For convenience, let us introduce some notations.

$$
N_{1}=\frac{1}{\Gamma(\alpha)}\left(\frac{1-q}{\alpha-q} b^{\frac{\alpha-q}{1-q}}\right)^{1-q}\|\Lambda\|_{L \frac{1}{q}(J, X)}, N_{2}=\frac{1}{\Gamma(\alpha)}\left\|y_{0}-x_{0}\right\| .
$$

Theorem 3. Suppose that hypotheses $\left(\mathcal{B}_{1}\right)$ and $\left(\mathscr{B}_{2}\right)$ are satisfied. If $K M b<1$, then problem (1.1) has at least one mild solution $x$ satisfying the estimates

$$
\|x-y\|_{C_{1-\alpha}} \leq M N_{2}+\frac{K M^{2} b\left(b^{1-\alpha} N_{1}+N_{2}\right)}{(1-K M b) \Gamma(\alpha+1)}+M N_{1} b^{1-\alpha} .
$$


Proof. We construct a sequence of functions $\left\{x_{n}\right\}_{n \in N}$ which will be shown to converge to some solution of problem (1.1) on the interval $[0, b]$, namely to

$$
\left\{\begin{array}{l}
{ }^{L} D^{\alpha} x(t) \in A x(t)+F(t, x(t)), \text { a.e. } t \in(0, b], 0<\alpha<1, \\
\left.I_{0^{+}}^{1-\alpha} x(t)\right|_{t=0}=x_{0} .
\end{array}\right.
$$

Let $f_{0}=g$ on $[0, b]$ and $x_{0}(t)=y(t), t \in(0, b]$, i.e.

$$
x_{0}(t)=t^{\alpha-1} T_{\alpha}(t) y_{0}+\int_{0}^{t}(t-s)^{\alpha-1} T_{\alpha}(t-s) f_{0}(s) d s, t \in(0, b] .
$$

Then define the multivalued map $U_{1}:[0, b] \rightarrow \mathcal{P}(X)$ by $U_{1}(t)=F\left(t, x_{0}(t)\right)$ $\cap B\left(f_{0}(t), \Lambda(t)\right)$. Since $g$ and $\Lambda$ are measurable, the ball $B\left(f_{0}(t), \Lambda(t)\right)$ is measurable (see [5]). Moreover $F\left(t, x_{0}(t)\right)$ is measurable (see [3]) and $U_{1}$ is nonempty. Indeed, according to Lemma 4, we obtain a measurable selection $u$ of $F\left(t, x_{0}(t)\right)$ such that

$$
\|u(t)-g(t)\| \leq d\left(g(t), F\left(t, x_{0}(t)\right)\right)=\Lambda(t) .
$$

Then $u(t) \in U_{1}(t)$, proving our claim. We deduce that the multivalued operator $U_{1}(t)$ is measurable $($ see $[3,5]) . X$ is a separable Banach space, by Lemma 2, there exists a function $t \rightarrow f_{1}(t)$ which is a measurable selection for $U_{1}$ and

$$
\left\|f_{1}(t)-f_{0}(t)\right\| \leq \Lambda(t) .
$$

Consider

$$
x_{1}(t)=t^{\alpha-1} T_{\alpha}(t) x_{0}+\int_{0}^{t}(t-s)^{\alpha-1} T_{\alpha}(t-s) f_{1}(s) d s, t \in(0, b] .
$$

For each $t \in[0, b]$, we have

$$
\begin{aligned}
& t^{1-\alpha}\left\|x_{1}(t)-x_{0}(t)\right\| \\
& =\left\|T_{\alpha}(t) x_{0}-T_{\alpha}(t) y_{0}\right\|+\left\|t^{1-\alpha} \int_{0}^{t}(t-s)^{\alpha-1} T_{\alpha}(t-s)\left[f_{1}(s)-f_{0}(s)\right] d s\right\| \\
& \leq \frac{M}{\Gamma(\alpha)}\left\|y_{0}-x_{0}\right\|+\frac{M t^{1-\alpha}}{\Gamma(\alpha)} \int_{0}^{t}(t-s)^{\alpha-1} \Lambda(s) d s \\
& \leq \frac{M}{\Gamma(\alpha)}\left\|y_{0}-x_{0}\right\|+\frac{M t^{1-\alpha}}{\Gamma(\alpha)}\left(\frac{1-q}{\alpha-q} b^{\frac{\alpha-q}{1-q}}\right)^{1-q}\|\Lambda\|_{L^{\frac{1}{q}}\left(J, R^{+}\right)} \\
& \leq M N_{2}+M N_{1} t^{1-\alpha} .
\end{aligned}
$$

Using the fact that $F\left(t, x_{1}(t)\right)$ is measurable, the ball $B\left(f_{1}(t), K t^{1-\alpha}\left\|x_{1}(t)-x_{0}(t)\right\|\right)$ is also measurable (see [5]). From $\left(\mathscr{B}_{2}\right)$ we have

$$
H_{d}\left(F\left(t, x_{1}(t)\right), F\left(t, x_{0}(t)\right)\right) \leq K t^{1-\alpha}\left\|x_{1}(t)-x_{0}(t)\right\| .
$$

Hence there exists $\omega \in F\left(t, x_{1}(t)\right)$ such that

$$
\left\|f_{1}(t)-\omega\right\| \leq K t^{1-\alpha}\left\|x_{1}(t)-x_{0}(t)\right\| .
$$


Consider the following multivalued map $U_{2}(t)=F\left(t, x_{1}(t)\right) \cap B\left(f_{1}(t), K t^{1-\alpha}\right.$ $\left.\left\|x_{1}(t)-x_{0}(t)\right\|\right)$, which is nonempty since it contains $\omega$. Therefore $U_{2}(t)$ is measurable with nonempty, closed values (see $[3,5])$. Thus there exists a measurable selection $f_{2}(t) \in U_{2}(t)$ and

$$
\left\|f_{2}(t)-f_{1}(t)\right\| \leq K t^{1-\alpha}\left\|x_{1}(t)-x_{0}(t)\right\| .
$$

Define

$$
x_{2}(t)=t^{\alpha-1} T_{\alpha}(t) x_{0}+\int_{0}^{t}(t-s)^{\alpha-1} T_{\alpha}(t-s) f_{2}(s) d s, t \in(0, b] .
$$

Then for every $t \in[0, b]$, we have

$$
\begin{aligned}
t^{1-\alpha}\left\|x_{2}(t)-x_{1}(t)\right\| & =\left\|t^{1-\alpha} \int_{0}^{t}(t-s)^{\alpha-1} T_{\alpha}(t-s)\left[f_{2}(s)-f_{1}(s)\right] d s\right\| \\
& \leq \frac{t^{1-\alpha} M K}{\Gamma(\alpha)} \int_{0}^{t}(t-s)^{\alpha-1}\left[M N_{1} s^{1-\alpha}+M N_{2}\right] d s \\
& \leq \frac{K M^{2} N_{1} t^{2-\alpha} \Gamma(2-\alpha)}{\Gamma(2)}+\frac{t M^{2} K N_{2}}{\Gamma(\alpha+1)} .
\end{aligned}
$$

Let $U_{3}(t)=F\left(t, x_{2}(t)\right) \cap B\left(f_{2}(t), K t^{1-\alpha}\left\|x_{2}(t)-x_{1}(t)\right\|\right)$. Arguing as for $U_{2}$, we can prove that $U_{3}$ is a measurable multivalued map with nonempty closed values. Then there exists a measurable selection $f_{3}(t) \in U_{3}(t)$ and

$$
\left\|f_{3}(t)-f_{2}(t)\right\| \leq K t^{1-\alpha}\left\|x_{2}(t)-x_{1}(t)\right\| .
$$

Define

$$
x_{3}(t)=t^{\alpha-1} T_{\alpha}(t) x_{0}+\int_{0}^{t}(t-s)^{\alpha-1} T_{\alpha}(t-s) f_{3}(s) d s, t \in(0, b] .
$$

For $t \in[0, b]$, we have

$$
\begin{aligned}
& t^{1-\alpha}\left\|x_{3}(t)-x_{2}(t)\right\| \\
& =\left\|t^{1-\alpha} \int_{0}^{t}(t-s)^{\alpha-1} T_{\alpha}(t-s)\left[f_{3}(s)-f_{2}(s)\right] d s\right\| \\
& \leq \frac{t^{1-\alpha} M K}{\Gamma(\alpha)} \int_{0}^{t}(t-s)^{\alpha-1}\left[\frac{K M^{2} N_{1} s^{2-\alpha} \Gamma(2-\alpha)}{\Gamma(2)}+\frac{s M^{2} K N_{2}}{\Gamma(\alpha+1)}\right] d s \\
& \leq \frac{t^{3-\alpha} M^{3} K^{2} N_{1} \Gamma(2-\alpha) \Gamma(3-\alpha)}{\Gamma(2) \Gamma(3)}+\frac{t^{2} M^{3} K^{2} N_{2} \Gamma(2)}{\Gamma(\alpha+1) \Gamma(\alpha+2)} .
\end{aligned}
$$

Repeating the process for $n=0,1,2,3, \ldots$, we have

$$
\begin{aligned}
t^{1-\alpha}\left\|x_{n}(t)-x_{n-1}(t)\right\| \leq & \frac{K^{n-1} M^{n} t^{n-\alpha} N_{1} \Gamma(2-\alpha) \Gamma(3-\alpha) \ldots \Gamma(n-\alpha)}{\Gamma(2) \Gamma(3) \ldots \Gamma(n)} \\
& +\frac{K^{n-1} M^{n} t^{n-1} N_{2} \Gamma(2) \Gamma(3) \ldots \Gamma(n-1)}{\Gamma(\alpha+1) \Gamma(\alpha+2) \ldots \Gamma(\alpha+n-1)} .
\end{aligned}
$$


By induction, suppose that (5.1) holds for some $n$ and check (5.1) for $n+1$. Let $U_{n+1}(t)=F\left(t, x_{n}(t)\right) \cap B\left(f_{n}, K t^{1-\alpha}\left\|x_{n}(t)-x_{n-1}(t)\right\|\right)$. Since $U_{n+1}$ is a measurable multivalued map with nonempty closed values, there exists a measurable selection $f_{n+1}(t) \in U_{n+1}(t)$ and

$$
\left\|f_{n+1}(t)-f_{n}(t)\right\| \leq K t^{1-\alpha}\left\|x_{n}(t)-x_{n-1}(t)\right\|,
$$

which allows us to define

$$
x_{n+1}(t)=t^{\alpha-1} T_{\alpha}(t) x_{0}+\int_{0}^{t}(t-s)^{\alpha-1} T_{\alpha}(t-s) f_{n+1}(s) d s, t \in(0, b] .
$$

Then, for a.e. $t \in[0, b]$, we have

$$
\begin{aligned}
t^{1-\alpha} & \left\|x_{n+1}(t)-x_{n}(t)\right\| \\
= & \left\|t^{1-\alpha} \int_{0}^{t}(t-s)^{\alpha-1} T_{\alpha}(t-s)\left[f_{n+1}(s)-f_{n}(s)\right] d s\right\| \\
\leq & \frac{t^{1-\alpha} M K}{\Gamma(\alpha)} \int_{0}^{t}(t-s)^{\alpha-1}\left[\frac{K^{n-1} M^{n} s^{n-\alpha} N_{1} \Gamma(2-\alpha) \Gamma(3-\alpha) \ldots \Gamma(n-\alpha)}{\Gamma(2) \Gamma(3) \ldots \Gamma(n)}\right] d s \\
& +\frac{t^{1-\alpha} M K}{\Gamma(\alpha)} \int_{0}^{t}(t-s)^{\alpha-1}\left[\frac{K^{n-1} M^{n} s^{n-1} N_{2} \Gamma(2) \Gamma(3) \ldots \Gamma(n-1)}{\Gamma(\alpha+1) \Gamma(\alpha+2) \ldots \Gamma(\alpha+n-1)}\right] d s \\
\leq & \frac{K^{n} M^{n+1} t^{n+1-\alpha} N_{1} \Gamma(2-\alpha) \Gamma(3-\alpha) \ldots \Gamma(n+1-\alpha)}{\Gamma(2) \Gamma(3) \ldots \Gamma(n+1)} \\
& +\frac{K^{n} M^{n+1} t^{n} N_{2} \Gamma(2) \Gamma(3) \ldots \Gamma(n)}{\Gamma(\alpha+1) \Gamma(\alpha+2) \ldots \Gamma(\alpha+n)} .
\end{aligned}
$$

Consequently, (5.2) holds true for all $n \in N$. According to (5.2), we have

$$
t^{1-\alpha}\left\|x_{n+1}(t)-x_{n}(t)\right\| \leq K^{n} M^{n+1} b^{n+1-\alpha} N_{1}+K^{n} M^{n+1} b^{n} N_{2} .
$$

Since $K M b<1$, we infer that $\left\{x_{n}\right\}_{n \in N}$ is a cauchy sequence in $C_{1-\alpha}(J, X)$, converging uniformly to a limit function $x \in C_{1-\alpha}(J, X)$. Hence there exists a constant $L>0$, such that $\left\|x_{n}\right\|_{C_{1-\alpha}} \leq L$. Since $f_{n}(t) \in F\left(t, x_{n-1}(t)\right)$, from $\mathscr{B}_{1}(b)$ we have

$$
\left\|f_{n}(t)\right\| \leq \sigma(t)+k t^{1-\alpha}\left\|x_{n-1}(t)\right\| \leq \sigma(t)+k\left\|x_{n-1}\right\|_{C_{1-\alpha}} \leq \sigma(t)+k L,
$$

which means that $\left\{f_{n}\right\}_{n \in N}$ is uniformly bounded in $L^{\frac{1}{q}}(J, X)$ and hence weakly converges to $f$ in $L^{\frac{1}{q}}(J, X)$. Let

$$
x(t)=t^{\alpha-1} T_{\alpha}(t) x_{0}+\int_{0}^{t}(t-s)^{\alpha-1} T_{\alpha}(t-s) f(s) d s, t \in(0, b] .
$$

Then we have

$$
\left\|x_{n}-x\right\|_{C_{1-\alpha}}=\sup _{t \in J}\left\|t^{1-\alpha} \int_{0}^{t}(t-s)^{\alpha-1} T_{\alpha}(t-s)\left[f_{n}(s)-f(s)\right] d s\right\|
$$




$$
\leq b^{1-\alpha} \sup _{t \in J}\left\|\int_{0}^{t}(t-s)^{\alpha-1} T_{\alpha}(t-s)\left[f_{n}(s)-f(s)\right] d s\right\| .
$$

Noting that the mapping $g \rightarrow \int_{0}^{\cdot}(\cdot-s)^{\alpha-1} T_{\alpha}(\cdot-s) g(s) d s: L^{\frac{1}{q}}(J, X) \rightarrow C(J, X)$ is compact, then $\left\|x_{n}-x\right\|_{C_{1-\alpha}} \rightarrow 0$, as $n \rightarrow \infty$. We find that the function $x$ is a solution to problem $(1.1)$ on $[0, b]$. Moreover, we have

$$
\begin{aligned}
t^{1-\alpha}\|x(t)-y(t)\|= & \left\|T_{\alpha}(t) x_{0}-T_{\alpha}(t) y_{0}\right\| \\
& +\left\|t^{1-\alpha} \int_{0}^{t}(t-s)^{\alpha-1} T_{\alpha}(t-s)[f(s)-g(s)] d s\right\| \\
\leq & M N_{2}+\left\|t^{1-\alpha} \int_{0}^{t}(t-s)^{\alpha-1} T_{\alpha}(t-s)\left[f(s)-f_{n}(s)\right] d s\right\| \\
& +\left\|t^{1-\alpha} \int_{0}^{t}(t-s)^{\alpha-1} T_{\alpha}(t-s)\left[f_{n}(s)-g(s)\right] d s\right\| .
\end{aligned}
$$

Noting that

$$
\begin{aligned}
\left\|f_{n}(t)-g(t)\right\| & \leq \sum_{i=1}^{n}\left\|f_{i}(t)-f_{i-1}(t)\right\| \\
& \leq \sum_{i=2}^{n} K t^{1-\alpha}\left\|x_{i-1}(t)-x_{i-2}(t)\right\|+\Lambda(t) \\
& \leq \sum_{i=1}^{\infty} K t^{1-\alpha}\left\|x_{i}(t)-x_{i-1}(t)\right\|+\Lambda(t) \\
& \leq \sum_{i=1}^{\infty}\left[K^{i} M^{i} b^{i-\alpha} N_{1}+K^{i} M^{i} b^{i-1} N_{2}\right]+\Lambda(t) \\
& \leq \frac{K M\left(b^{1-\alpha} N_{1}+N_{2}\right)}{1-K M b}+\Lambda(t),
\end{aligned}
$$

we have

$$
\begin{aligned}
t^{1-\alpha}\|x(t)-y(t)\| \leq & M N_{2}+\left\|t^{1-\alpha} \int_{0}^{t}(t-s)^{\alpha-1} T_{\alpha}(t-s)\left[f(s)-f_{n}(s)\right] d s\right\| \\
& +\left\|t^{1-\alpha} \int_{0}^{t}(t-s)^{\alpha-1} T_{\alpha}(t-s)\left[f_{n}(s)-g(s)\right] d s\right\| \\
\leq & M N_{2}+\frac{K M^{2} b\left(b^{1-\alpha} N_{1}+N_{2}\right)}{(1-K M b) \Gamma(\alpha+1)}+M N_{1} b^{1-\alpha}, n \rightarrow \infty .
\end{aligned}
$$

Then, we have

$$
\|x-y\|_{C_{1-\alpha}} \leq M N_{2}+\frac{K M^{2} b\left(b^{1-\alpha} N_{1}+N_{2}\right)}{(1-K M b) \Gamma(\alpha+1)}+M N_{1} b^{1-\alpha} .
$$


The proof is complete.

\section{AN EXAMPLE}

Let $X=L^{2}([0, \pi], R)$. As an application of our results, consider the following fractional partial differential inclusions.

$$
\left\{\begin{array}{l}
{ }^{L} D_{0+}^{\frac{3}{4}} x(t, y) \in \frac{\partial^{2}}{\partial y^{2}} x(t, y)+F(t, x(t, y)), \text { a.e. } t \in(0, b], y \in[0, \pi], \\
x(t, 0)=x(t, \pi)=0, t \in(0, b], \\
\left.I_{0^{+}}^{\frac{1}{4}} x(t, y)\right|_{t=0}=x_{0}(y), y \in[0, \pi] .
\end{array}\right.
$$

where ${ }^{L} D_{0+}^{\frac{3}{4}}$ is the Riemann-Liouville fractional derivative of order $\frac{3}{4}, b>0, I_{0^{+}}^{\frac{1}{4}}$ is Riemann-Liouville integral of order $\frac{1}{4}, J=[0, b]$. $F$ is a given multivalued map. Define the operator $A$ by $A x=x^{\prime \prime}$, with the domain

$D(A)=\left\{x \in X: x, x^{\prime}\right.$ are absolutely continuous, $\left.x^{\prime \prime} \in X, x(t, 0)=x(t, \pi)=0\right\}$.

Then $A$ can be written as

$$
A x=-\sum_{n=1}^{\infty} n^{2}<x, e_{n}>e_{n}, x \in D(A),
$$

where $e_{n}(y)=\sqrt{\frac{2}{\pi}} \sin n y, 0 \leq y \leq \pi, n=1,2, \ldots$ is an orthonormal basis of $X$. It is well known that $A$ is the infinitesimal generator of a strongly continuous semigroup $T(t)$ on $X$ which is compact, given by

$$
T(t) x=\sum_{n=1}^{\infty} e^{-n^{2} t}<x, e_{n}>e_{n}, x \in X, \text { and }\|T(t)\| \leq e^{-t} \leq 1=M, t \geq 0 .
$$

Define $x(t, y)=x(t)(y)$, then system (6.1) can be written in the abstract form given by (1.1). We assume that $F: J \times X \rightarrow \mathcal{P}(X)$ satisfy the following conditions.

$\left(F_{1}\right): F: J \times X \rightarrow \mathcal{P}_{c p, c v}(X)$ is measurable to $t$ for each fixed $u \in X$, u.s.c. to $u$ for a.e. $t \in J$, and for each $x \in C_{\frac{1}{4}}(J, X)$ the set

$$
S_{F, x}=\left\{f \in L^{1}(J, X): f(t) \in F(t, x(t))\right\}
$$

is nonempty.

$\left(F_{2}\right)$ : There exists a function $\phi(t) \in L^{\frac{1}{q}}\left(J, R^{+}\right), q \in\left(0, \frac{3}{4}\right)$ and a constant $c>0$ such that

$$
\|F(t, x(t))\|_{X}=\sup \left\{\|f(t)\|_{X}: f(t) \in F(t, x(t))\right\} \leq \phi(t)+c t^{\frac{1}{4}}\|x(t)\|,
$$

for a.e. $t \in J$ and all $x \in C_{\frac{1}{4}}(J, X)$.

Thus by Theorem 1, system (6.1) has at least one mild solution. 


\section{REFERENCES}

[1] B. Ahmad and S. K. Ntouyas, "Boundary value problems for $n$-th order differential inclusions with four-point integral boundary conditions," Opuscula Mathematica, vol. 32, no. 2, pp. 205226, 2012.

[2] J. P. Aubin and A. Cellina, Differential inclusions: set-valued maps and viability theory. Springer-Verlag New York, Inc., 1984.

[3] J.-P. Aubin and H. Frankowska, "Set-valued analysis. 1990,” Birhäuser, Boston, 1965.

[4] M. Benchohra, J. Henderson, and S. Ntouyas, "On nonresonance second order impulsive functional differential inclusions with nonlinear boundary conditions," Canadian Appl. Math. Quart.(to appear), 2006.

[5] C. Castaing and M. Valadier, Convex analysis and measurable multifunctions. Springer, 1977.

[6] H. Covitz and S. Nadler, "Multi-valued contraction mappings in generalized metric spaces," Israel Journal of Mathematics, vol. 8, no. 1, pp. 5-11, 1970, doi: 10.1007/BF02771543.

[7] K. Deimling, Multivalued differential equations. Walter de Gruyter, 1992, vol. 1.

[8] L. Górniewicz, Topological fixed point theory of multivalued mappings. Springer, 2006, vol. 4, doi: 10.1007/1-4020-4666-9.

[9] A. Granas and J. Dugundji, Fixed point theory. Springer, 2003.

[10] J. Henderson and A. Ouahab, "Fractional functional differential inclusions with finite delay," Nonlinear Analysis: Theory, Methods \& Applications, vol. 70, no. 5, pp. 2091-2105, 2009, doi: 10.1016/j.na.2008.02.111.

[11] D. Henry, Geometric Theory of Semilinear Parabolic Equations, ser. Lecture Notes in Mathematics. Springer Berlin Heidelberg, 1993.

[12] S. Hu and N. Papageorgiou, "Handbook of multivalued analysis. theory, vol. I," 1997.

[13] A. A. A. Kilbas, H. M. Srivastava, and J. J. Trujillo, Theory and applications of fractional differential equations. Elsevier Science Limited, 2006, vol. 204.

[14] A. Kilbas, O. Marichev, and S. Samko, "Fractional integral and derivatives (theory and applications)," Gordon and Breach, Switzerland, vol. 1, no. 993, p. 1, 1993.

[15] M. Kisielewicz, Differential inclusions and optimal control. Kluwer Academic Dordrecht, 1991.

[16] A. Lasota and Z. Opial, "An application of the Kakutani-Ky Fan theorem in the theory of ordinary differential equations,” Bull. Acad. Polon. Sci. Ser. Sci. Math. Astronom. Phys., vol. 13, pp. 781$786,1965$.

[17] X. Liu and Z. Liu, "Existence results for a class of second order evolution inclusions and its corresponding first order evolution inclusions," Israel Journal of Mathematics, vol. 194, no. 2, pp. 723-743, 2013, doi: 10.1007/s11856-012-0092-2.

[18] Z. Liu and S. Migórski, "Analysis and control of differential inclusions with anti-periodic conditions," Proceedings of the Royal Society of Edinburgh: Section A Mathematics, vol. 144, no. 03, pp. 591-602, 2014.

[19] Z. Liu and X. Li, "On the controllability of impulsive fractional evolution inclusions in Banach spaces," Journal of Optimization Theory and Applications, vol. 156, no. 1, pp. 167-182, 2013, doi: 10.1007/s10957-012-0236-x.

[20] K. S. Miller and B. Ross, An introduction to the fractional calculus and fractional differential equations. John Wiley \& Sons, 1993.

[21] A. Ouahab, "Fractional semilinear differential inclusions," Computers \& Mathematics with Applications, vol. 64, no. 10, pp. 3235-3252, 2012, doi: 10.1016/j.camwa.2012.03.039.

[22] I. Podlubny, Fractional differential equations: an introduction to fractional derivatives, fractional differential equations, to methods of their solution and some of their applications. Academic press, 1998, vol. 198. 
[23] J. Wang and Y. Zhou, "Existence and controllability results for fractional semilinear differential inclusions," Nonlinear Analysis: Real World Applications, vol. 12, no. 6, pp. 3642-3653, 2011, doi: 10.1016/j.nonrwa.2011.06.021.

[24] Y. Zhou and F. Jiao, "Existence of mild solutions for fractional neutral evolution equations," Computers \& Mathematics with Applications, vol. 59, no. 3, pp. 1063-1077, 2010, doi: 10.1016/j.camwa.2009.06.026.

[25] Y. Zhou, L. Zhang, X. H. Shen et al., "Existence of mild solutions for fractional evolution equations," Journal of Integral Equations and Applications, vol. 25, no. 4, pp. 557-586, 2013, doi: 10.1216/JIE-2013-25-4-557.

[26] Q. J. Zhu, "On the solution set of differential inclusions in Banach space," Journal of Differential Equations, vol. 93, no. 2, pp. 213-237, 1991, doi: 10.1016/0022-0396(91)90011-W.

Authors' addresses

\section{Yong Huang}

Department of Mathematics, Baise University, Baise 533000, Guangxi Province, P.R. China

E-mail address: yonghuang99@126.com

Jingyun Lv

Guangxi Key Laboratory of Universities Optimization Control and Engineering Calculation, and College of Science, Guangxi University for Nationalities, Nanning, Guangxi 530006, P.R. China E-mail address: lvjingyun2007@163.com

\section{Zhenhai Liu}

Guangxi Key Laboratory of Universities Optimization Control and Engineering Calculation, and College of Science, Guangxi University for Nationalities, Nanning, Guangxi 530006, P.R. China E-mail address: zhhliu@hotmail.com 